\title{
Near-Transform-Limited Single Photons from an Efficient Solid-State Quantum Emitter
}

\author{
Hui Wang, ${ }^{1,2,3}$ Z.-C. Duan, ${ }^{1,2,3}$ Y.-H. Li, ${ }^{1,2,3}$ Si Chen, ${ }^{1,2,3}$ J.-P. Li, ${ }^{1,2,3}$ Y.-M. He, ${ }^{1,4}$ M.-C. Chen, ${ }^{1,2,3}$ \\ Yu He,${ }^{1,2,3}$ X. Ding, ${ }^{1,2,3}$ Cheng-Zhi Peng, ${ }^{1,2,3}$ Christian Schneider, ${ }^{4}$ Martin Kamp, ${ }^{4}$ Sven Höfling, ${ }^{1,4,5}$ \\ Chao-Yang Lu, ${ }^{1,2,3, *}$ and Jian-Wei Pan ${ }^{1,2,3, \dagger}$ \\ ${ }^{1}$ Shanghai Branch, National Laboratory for Physical Sciences at Microscale and Department of Modern Physics, \\ University of Science and Technology of China, Shanghai 201315, China \\ ${ }^{2}$ CAS Center for Excellence and Synergetic Innovation Center in Quantum Information and Quantum Physics, \\ University of Science and Technology of China, Hefei, Anhui 230026, China \\ ${ }^{3}$ CAS-Alibaba Quantum Computing Laboratory, Shanghai 201315, China \\ ${ }^{4}$ Technische Physik, Physikalisches Instität and Wilhelm Conrad Röntgen-Center for Complex Material Systems, \\ Universitat Würzburg, Am Hubland, D-97074 Wüzburg, Germany \\ ${ }^{5}$ SUPA, School of Physics and Astronomy, University of St. Andrews, St. Andrews KY16 9SS, United Kingdom
}

(Received 19 February 2016; published 27 May 2016)

\begin{abstract}
By pulsed $s$-shell resonant excitation of a single quantum dot-micropillar system, we generate long streams of 1000 near-transform-limited single photons with high mutual indistinguishability. The HongOu-Mandel interference of two photons is measured as a function of their emission time separation varying from $13 \mathrm{~ns}$ to $14.7 \mu \mathrm{s}$, where the visibility slightly drops from $95.9(2) \%$ to a plateau of $92.1(5) \%$ through a slow dephasing process occurring at a time scale of $0.7 \mu \mathrm{s}$. A temporal and spectral analysis reveals the pulsed resonance fluorescence single photons are close to the transform limit, which are readily useful for multiphoton entanglement and interferometry experiments.
\end{abstract}

DOI: 10.1103/PhysRevLett.116.213601

Self-assembled InGaAs quantum dots (QDs) are promising single-photon emitters with a high quantum efficiency and a fast decay rate [1]. In the past decades, extensive efforts have been devoted to producing single photons with high purity (that is, a vanishing two-photon emission probability), near-unity indistinguishability, and high extraction efficiency [2-10]. These key properties have been compatibly combined simultaneously on the same QD micropillar very recently [11-13].

An important next challenge is to extend the singlephoton sources to multiple photonic quantum bits [14], as required by various quantum information protocols such as boson sampling [15], quantum teleportation [16], quantum computation [17], and quantum metrology [18]. To this aim, one approach is to use many independent QDs [19] that are tuned into an identical emission wavelength [20] and efficiently emit single photons stringently at the transform limit, that is, $T_{2}=2 T_{1}$, where $T_{2}$ and $T_{1}$ are the photon's coherence time and lifetime, respectively. Another-probably less demanding-solution is based on only one perfect QD emitting single-photon pulse trains with high efficiency [11,12], which are then either demultiplexed into $N$ spatial modes or dynamically controlled using time-bin encoding in a loop-based architecture [21]. Implementing $N$-photon quantum circuits in this configuration demands streams of $N$ mutually indistinguishable single photons far apart in emission time.

However, previous Hong-Ou-Mandel (HOM) type interference experiments [7-13] were performed with a time separation of only a few nanoseconds between two photons emitted consecutively from a QD. Spectral diffusions [22] with a time scale much slower than nanoseconds were speculated-yet without a conclusive study-to account for the mismatch between the observed near-unity transient indistinguishability and the nonunity time-averaged $T_{2} / 2 T_{1}$ ratio [7-10,13]. Thus, it is highly desirable to study the two-photon interference as a function of their emission time separation and test how far apart the high indistinguishability persists. The ultimate goal is to generate efficient and truly transform-limited single photons, with which perfect interference can be achieved regardless of their time separation, and even if the photons are from independent QDs.

An interesting remedy [23] to circumvent the environment-induced dephasing is to operate in the small (typically $<10 \%$ ) Rabi frequency regime of resonance fluorescence (RF) [24]. Under weak excitation, indistinguishable single photons with a subnatural linewidth and phase locked to the excitation laser have been probabilistically generated [23], which has found applications in solid-state quantum networking [25]. Another work revealed signatures of a near-transform-limited optical linewidth using resonant laser spectroscopy [26], yet without a direct measurement of the emitted photons. Importantly, in these works $[23,26]$, the photon generation and collection efficiencies were intrinsically limited due to both the sample structures and the nondeterministic continuous-wave excitation. More recently, the nonresonant pumping of QDs grown by metal-organic chemical vapor deposition has generated single photon streams whose mutual indistinguishability 
decreases rapidly from $94(6) \%$ at 2 ns separation to $53(3) \%$ at $12.5 \mathrm{~ns}$ separation [27].

In this Letter, we demonstrate for the first time that a pulsed resonantly driven single QD embedded in a micropillar emits long streams of at least 1000 single photons with high (>90\%) mutual indistinguishability, showing its promise for multiphoton experiments. Time-dependent HOM experiments reveal an indistinguishability of 95.9(2)\% for two single photons separated by $13 \mathrm{~ns}$, which decreases to a plateau of $92.1(5) \%$ at $\sim 2-14.7 \mu$ s separation, through a dephasing process occurring at a submicrosecond time scale. Temporal and spectral measurements further confirm the single photons are close to the transform limit: $T_{2} / 2 T_{1}=0.91(5)$.

Our experimental arrangement is shown in Fig. 1(a). A single InAs/GaAs self-assembled QD embedded inside a $2 \mu \mathrm{m}$ diameter micropillar cavity (for more details on the sample growth and fabrication, see Ref. [28]) is cooled to 4.3 $\mathrm{K}$ where the QD emission is resonant with the cavity mode [see Fig. 1(b)]. The QD micropillar is excited by a resonant, picosecond laser (see Ref. [11] for details), allowing a clear observation of the Rabi oscillation in the detected RF photons as a function of laser field strength. At a $\pi$ pulse with a repetition rate of $76.4 \mathrm{MHz}$, the QD micropillar emits $\sim 5 \times 10^{6}$ pulsed RF single photons at the output of a single-mode fiber (absolute source brightness $\sim 6.6 \%$ ), of which $1.67 \times 10^{6}$ are finally detected by a silicon single-photon detector. The single-photon nature of the generated pulsed RF is unambiguously proven from intensity-correlation measurements [29] [see Fig. 2(d)], which show that at zero delay the multiphoton probability is almost vanishing $\left[g^{2}(0)=0.007(1)\right]$.

The main result of this experiment is to measure the indistinguishability of the pulsed RF single photons as a function of their emission time separation $\Delta$, and test how far apart two photons can be and still remain indistinguishable. As shown in Fig. 1(a), the single photons are fed into an unbalanced Mach-Zehnder interferometer with its path length difference variable using $2 \mathrm{~m}$ to $3 \mathrm{~km}$ optical fibers. We first test the HOM interference between two consecutively emitted single photons at a time delay of $13 \mathrm{~ns}$ - the laser pulse separation. If two identical photons are combined on a beam splitter, they will always exit the beam splitter together through the same output port, a unique quantum phenomenon that cannot be explained by classical optics [32]. Figure 2(a) shows the time-delayed histograms of normalized twophoton coincidence counts for cross (black) and parallel (red) polarization. A significant suppression of the counts is observed at zero delay when the two incoming photons are prepared in the parallel polarization state. We obtain a degree of indistinguishability of $0.959(2)$ between the two $\pi$-pulse excited single photons separated by $13 \mathrm{~ns}$. Reducing the laser excitation power can yield a slightly higher indistinguishability up to $\sim 0.982(3)$ (see the (a)
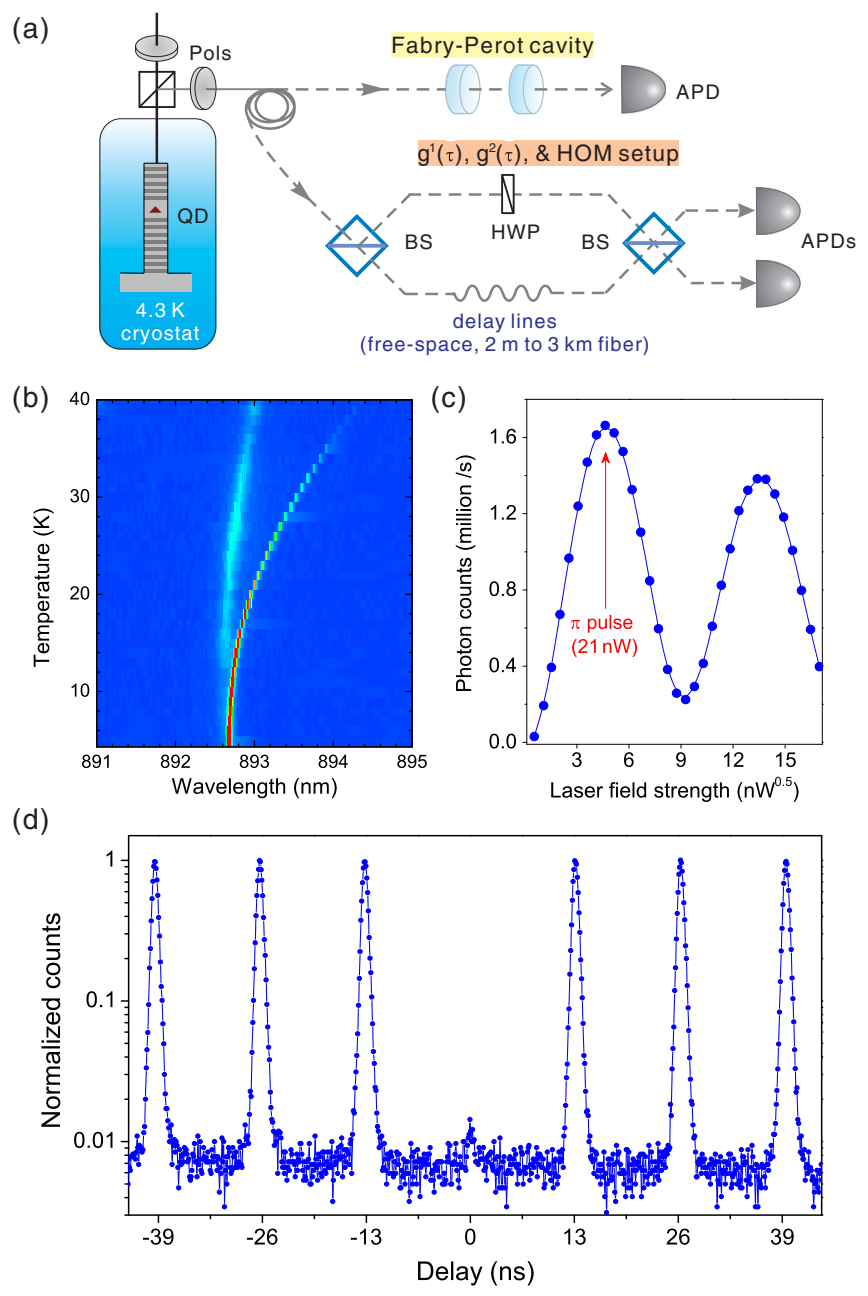

FIG. 1. Generation and characterization of single photons from a QD embedded in a micropillar. (a) An illustration of the experimental setup. The QD is grown by molecular beam epitaxy and sandwiched between 25.5 (15) lower (upper) distributed Bragg reflectors stacks. Pillars with a diameter of $2 \mu \mathrm{m}$ are defined via electron beam lithography [30]. The quality factor of the micropillar cavity is measured to be 5349 (see the Supplemental Material, Fig. S1 [[31]]). The emitted RF single photons are sent to a Fabry-Perot cavity for high-resolution spectral analysis, or to an unbalanced Mach-Zehnder interferometer for $g^{1}(\tau), g^{2}(\tau)$, and HOM measurements. (b) Temperature-dependent 2D intensity plot of the QD photoluminescence and the cavity mode. The excitation laser is at $780 \mathrm{~nm}$ with a power of $\sim 0.3 \mathrm{nW}$. (c) Detected single photon counts as a function of the pump field strength. (d) Intensity-correlation histogram of the RF photons. The measured second-order correlation at zero delay is $g^{2}(0)=0.007(1)$.

Supplemental Material, Fig. S2 [[31]]), possibly due to a decrease of excitation induced dephasing [33].

Similar experiments are performed by increasing the time separation to $289 \mathrm{~ns}, 0.83 \mu \mathrm{s}, 1.67 \mu \mathrm{s}, 5.11 \mu \mathrm{s}$, and $14.7 \mu \mathrm{s}$, which is $3-4$ orders of magnitude longer than the previous work $[7,8,11,12,27]$. The measurement result for the furthest separation of $14.7 \mu \mathrm{s}$ - corresponding to the HOM interference between the $n$th and the $(n+1120)$ th photon in the pulsed train-is plotted in 

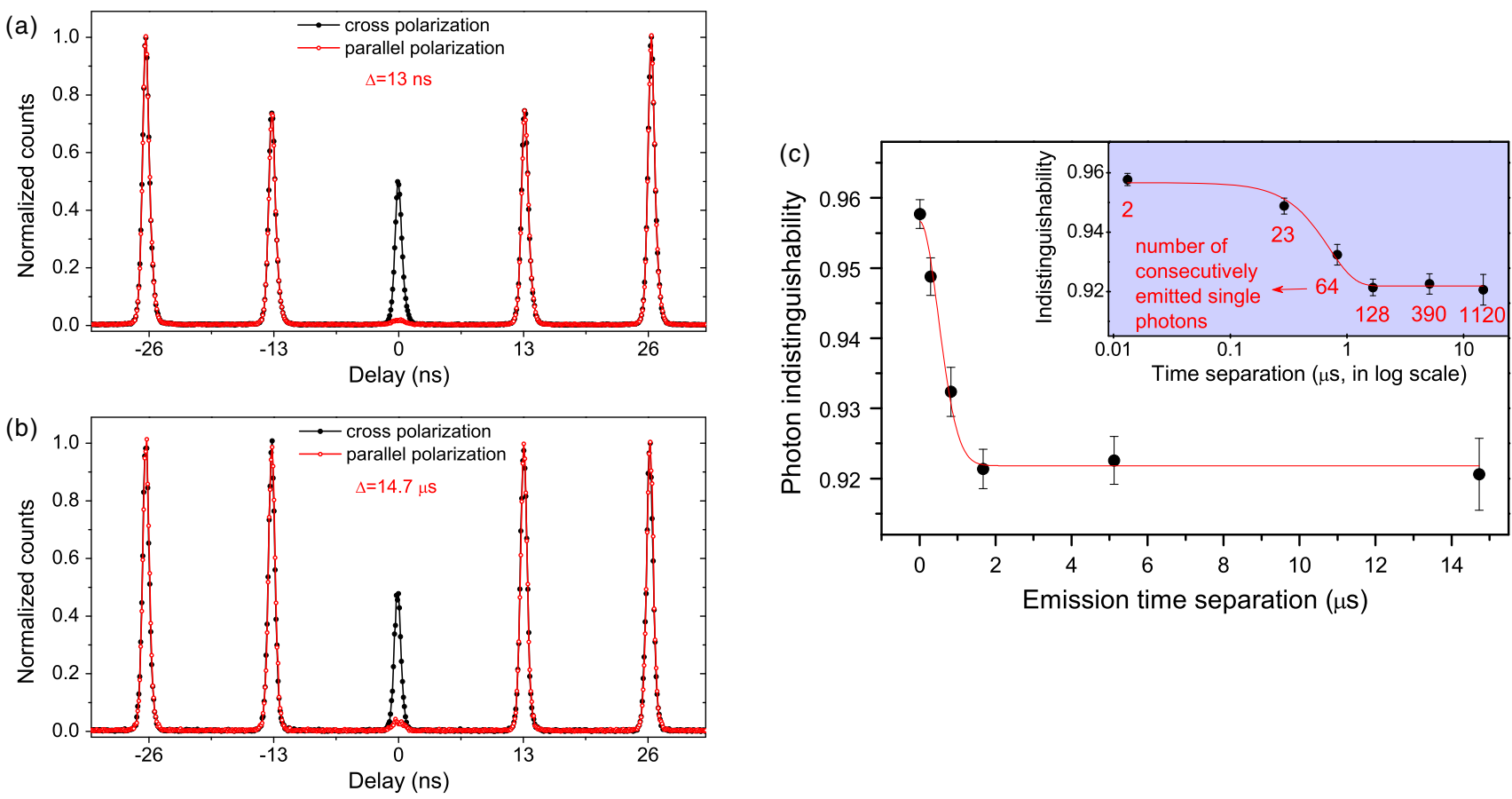

FIG. 2. Single photon indistinguishability measured from HOM experiments at various photon emission time separations $\Delta$. (a) $\Delta=13 \mathrm{~ns}$. The input two photons are $\pi$-pulse excited and prepared in cross (black) and parallel (red) polarizations. The data accumulation time was $5 \mathrm{~min}$. The data points presented are raw data without background subtraction. (b) $\Delta=14.7 \mu \mathrm{s}$, while keeping all the other physical conditions the same as in (a). (c) The extracted photon indistinguishability as a function of emission time separation. The small window of a few percent difference in the indistinguishability is precisely measured with a small error bar, which is possible due to the high photon count rate (that eliminates the shot noise) from the efficient QD-micropillar device [11]. The same data are shown in the inset that plots the $x$ axis in log scale and highlights the number of consecutively emitted single photons for each data point. The red curve is a fit using the model derived in Ref. [27] assuming non-Markovian noise.

Fig. 2(b), which shows a photon indistinguishability of $0.921(5)$, only a small decrease from the figure of merit at $\Delta=13 \mathrm{~ns}$. This finding demonstrates that the high mutual indistinguishability can be sustained among at least 1000 single photons from a QD micropillar, making it particularly suitable for optical quantum computing protocols with time-bin encoding [21].

Figure 2(c) summaries the measured indistinguishability as a function of photon emission time separations, and the inset highlights the number of consecutively emitted single photons for each data point. The photon indistinguishability slightly drops from $95.9(2) \%$ at 13 ns to a plateau of $92.1(5) \%$ at a $\sim 2-14.7 \mu$ s time separation. The timedependent HOM measurements reveal a slow dephasing process occurring at a time scale of $\sim 0.7 \mu$ s, which is $\sim 4000$ times longer than the single-photon radiative decay lifetime. Such a dephasing process can be caused by spectral diffusion due to charge fluctuations in the vicinity of the QD [22].

We expect that two single photons emitted with a time separation much shorter than $0.7 \mu \mathrm{s}$ collectively feel the same environment; thus, their indistinguishability is immune to the slow spectral wandering, and thus near-unity visibilities were observed in the HOM measurements $[8,11,12]$. However, two photons separated much longer than microseconds experience a different electric field at their time of emission; therefore, their time averaged wave-packet overlap is determined by the amplitude of the spectral wandering compared to its intrinsic lifetimelimited linewidth.

Surprisingly, the photon indistinguishability maintains at a plateau of $92.1 \%$ in the long time separation regime, suggesting that the emitted single photons can be close to the transform limit. To confirm this, we measure both the lifetime and the coherence time of the single photons. As shown in Fig. 3(a), the photon lifetime at $4.3 \mathrm{~K}$ (black square) is measured to be $T_{1}=162(5) \mathrm{ps}$, which is shortened by a factor of 3.8 compared to the data at large detuning (red circle) due to the Purcell effect. We use the Mach-Zehnder interferometer to measure the coherence time of the single photons. The integration time for each data point is $0.02 \mathrm{~s}$ - a much longer time scale than those in the photon interference measurements. By fitting the MachZehnder interference visibilities as a function of temporal delay [see Fig. 3(b)], we extract the coherence time to be $T_{2}=294(8) \mathrm{ps}$, and thus $T_{2} / 2 T_{1}=0.91(5)$. This is in good agreement with the photon indistinguishability observed in the long time scale.

The emitted photons are further spectrally characterized by measuring their high-resolution spectra using a Fabry-Perot scanning cavity. The spectrum at $\pi$-pulse excitation is shown in Fig. 3(c) where each data point is integrated for $0.1 \mathrm{~s}$, which is again a slow measurement 

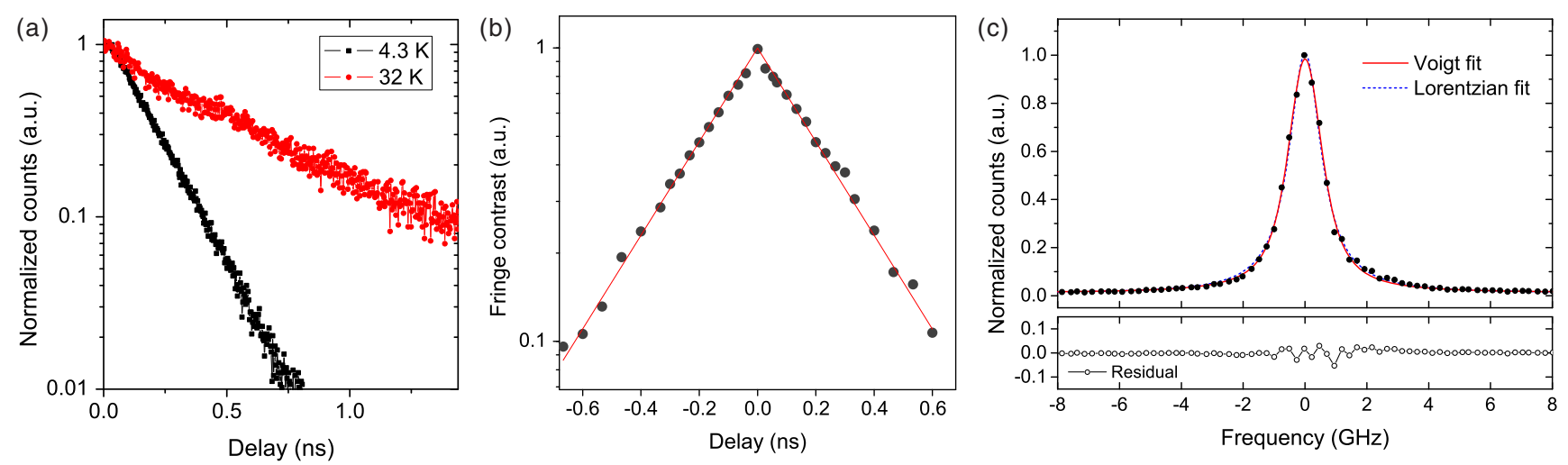

FIG. 3. Characterization of the single-photon source. (a) Time-resolved RF counts at QD-cavity resonance (4.3 K) and at far detuning (32 K). (b) Measurement of the coherence time of the single photons using a Mach-Zehnder interferometer, showing the fringe contrast versus path-length difference. The fringe contrast was calculated from registered single photon counts of one of the interferometer outputs while scanning one of the arm lengths over about 10 wavelengths. (c) A high-resolution RF spectrum when excited by a $\pi$ pulse, obtained using a home-built Fabry-Pérot scanning cavity with a finesse of 170, a linewidth of $220 \mathrm{MHz}$ (full width at half maximum), a free spectral range of $37.4 \mathrm{GHz}$, and a total transmission rate of $61 \%$. The blue line was a fit using a Lorentzian profile. The red line was a fit using a Voigt profile with the residual shown in the bottom panel. The Fourier transform of this spectrum after deconvolution with the 220-MHz Fabry-Pérot instrumental resolution gives a coherence time of 291(8) ps, in good agreement with (b).

compared to HOM interference. It can be slightly better fitted using a Voigt profile (red) than a pure Lorentzian (blue) profile, with a homogeneous (Lorentzian) and inhomogeneous (Gaussian) linewidth of 1.01(4) and 0.75(5) GHz, respectively. We expect that the Gaussian component could be due to inhomogeneous broadening caused by the spectral wandering around the center of the Lorentzian profile. Under this simple model, numerical analysis shows an average overlap of the photon wave packet of 0.90(2).

For the spectral measurement of our experiment, the Fabry-Perot scanning bandwidth is limited to $\sim 1 \mathrm{kHz}$ for a reasonable signal to noise ratio, due to the total photon counts. If the scanning frequency can be faster than $1 \mathrm{MHz}$, we would expect to see a narrowing of the spectrum and a transition from a Voigt to a purely Lorentzian profile.

The submicrosecond time scale of dephasing observed here is significantly longer than the previous results [9,13,27]; meanwhile, the spectral diffusion amplitude relative to the intrinsic lifetime-limited linewidth is smaller. Compared to the QDs grown by metal-organic vapor phase epitaxy, which is not carried out under ultrahigh vacuum conditions [27], the results suggest our sample grown by molecular beam epitaxy may have a smaller density of crystal impurities. Compared with the results that required stabilizing the electric environment around the QD with an additional laser [9] or electric gating [13], we conjecture that our QD-micropillar device in high purity GaAs is exposed to a less noisy environment, such as an etched surface, interface, or a crystal defect.

Controlled optical experiments are further performed to investigate the necessary condition for the generation of transform-limited single photons from this QD. We use nonresonant excitation on the same QD and test twophoton interference using the same HOM setup. First, we mix a small amount of $780 \mathrm{~nm}$ laser-typically used for above band gap excitation - into the $s$-shell excitation laser. The power of the $780 \mathrm{~nm}$ laser is on the order of tens of picowatts, which alone is too small to generate appreciable single photon counts. The nonresonant laser is filtered out in the output from the single photons using a long-pass filter. Figure S3 of the Supplemental Material [[31]] shows the dependence of the photon indistinguishability as a function of the amount of mixed nonresonant laser. We observe a gradual decrease of indistinguishability with an increasing amount of $780 \mathrm{~nm}$ laser, which goes down to $54 \%$ at $67 \mathrm{pW}$.

Second, we test $p$-shell excitation on the QD where the laser is tuned to $881 \mathrm{~nm}$. With a high pump power of $120 \mu \mathrm{W}$, the generated single photon rate is comparable to that under $21 \mathrm{nW} \pi$-pulse resonant excitation [Fig. 1(c)]. Under this condition, we measure the intensity correlation and photon indistinguishability. As shown in Fig. S4 of the Supplemental Material [[31]], while the source still exhibits a high single-photon purity, $g^{2}(0)=0.027(2)$, the interference visibility is measured to be $0.21(2)$, much lower than in $s$-shell excitation. The low visibility can be caused by high-power $(120 \mu \mathrm{W}$, as single-photon generation efficiencies increased asymptotically with pump power in incoherent excitation) laser excitation induced dephasing $[33,34]$, and the time jitter from the nonradiative $p$ - to $s$-shell incoherent relaxation [35]. These tests indicate that $s$-shell pulsed resonant excitation appears essential for the generation of transform-limited single photons on demand.

Finally, under strict resonant excitation, the photon indistinguishability is measured with the sample temperatures varying from 4.3 to $12 \mathrm{~K}$. It should be noted that the increase of temperature meanwhile brings the QD out of resonance with the micropillar cavity [see Fig. 1(b)]. The two-photon interference is tested with emission time separations of $13 \mathrm{~ns}$ and $14.7 \mu \mathrm{s}$. From the temperature-dependent results shown 
in Fig. S5 of the Supplemental Material [[31]], we observe an almost linear decrease of the indistinguishability with increasing temperature. This can be due to a combined effect of increased phonon-induced dephasing and reduced QD-cavity coupling [35-37]. This test suggests that QD-microcavity devices with a large Purcell enhancement at low temperature are desirable for emitting transform-limited single photons.

In summary, we have tested the time-dependent HOM interference between two single photons from a QD micropillar, with a time separation up to $14.7 \mu \mathrm{s}$, which not only elucidates the time dynamics of the dephasing process in single-photon generation, but also quantitatively reveals the noise amplitude from the spectral diffusion. Streams of 1000 mutually highly indistinguishable single photons are observed, lending them directly useful in boson sampling [38] and optical quantum computing algorithms using the time-bin encoding protocol [21]. Combining the temporal correlation measurements and the spectral analysis allows us to determine the single photons to be near transform limited $(>90 \%)$.

This work was supported by the National Natural Science Foundation of China, the Chinese Academy of Sciences, the National Fundamental Research Program, and the State of Bavaria.

Note added.-Recently, we became aware of a related paper [39].

*cylu@ustc.edu.cn †pan@ustc.edu.cn

[1] A. J. Shields, Nat. Photonics 1, 215 (2007); P. Lodahl, S. Mahmoodian, and S. Stobbe, Rev. Mod. Phys. 87, 347 (2015).

[2] B. Lounis and M. Orrit, Rep. Prog. Phys. 68, 1129 (2005); S. Buckley, K. Rivoire, and J. Vuĉković, Rep. Prog. Phys. 75, 126503 (2012).

[3] P. Michler et al., Science 290, 2282 (2000); C. Santori, M. Pelton, G. Solomon, Y. Dale, and Y. Yamamoto, Phys. Rev. Lett. 86, 1502 (2001).

[4] J. M. Gerard, B. Sermage, B. Gayral, B. Legrand, E. Costard, and V. Thierry-Mieg, Phys. Rev. Lett. 81, 1110 (1998); M. Pelton, C. Santori, J. Vuckovic, B. Zhang, G. S. Solomon, J. Plant, and Y. Yamamoto, Phys. Rev. Lett. 89, 233602 (2002); S. Strauf, N. G. Stoltz, M. T. Rakher, L. A. Coldren, P. M. Petroff, and D. Bouwmeester, Nat. Photonics 1, 704 (2007).

[5] D. Englund, D. Fattal, E. Waks, G. Solomon, B. Zhang, T. Nakaoka, Y. Arakawa, Y. Yamamoto, and J. Vuckovic, Phys. Rev. Lett. 95, 013904 (2005); K. H. Madsen, S. Ates, J. Liu, A. Javadi, S. M. Albrecht, I. Yeo, S. Stobbe, and P. Lodahl, Phys. Rev. B 90, 155303 (2014); L. Sapienza, M. Davanco, A. Badolata, and K. Srinivasan, Nat. Commun. 6, 7833 (2015).

[6] J. Claudon et al., Nat. Photonics 4, 174 (2010); M. E. Reimer, G. Bulgarini, N. Akopian, M. Hocevar, M. B. Bavinck, M. A. Verheijen, E. P. A. M. Bakkers, L. P. Kouwenhoven, and V. Zwiller, Nat. Commun. 3, 737 (2012).
[7] C. Santori, D. Fattal, J. Vučković, G. S. Solomon, and Y. Yamamoto, Nature (London) 419, 594 (2002); S. Ates, S. M. Ulrich, S. Reitzenstein, A. Loffler, A. Forchel, and P. Michler, Phys. Rev. Lett. 103, 167402 (2009); S. Unsleber, D. P. S. McCutcheon, M. Dambach, M. Lermer, N. Gregersen, S. Hofling, J. Mork, C. Schneider, and M. Kamp, Phys. Rev. B 91, 075413 (2015).

[8] Y.-M. He, Y. He, Y.-J. Wei, D. Wu, M. Atatüre, C. Schneider, S. Höfling, M. Kamp, C.-Y. Lu, and J.-W. Pan, Nat. Nanotechnol. 8, 213 (2013).

[9] O. Gazzano, S. Michaelis de Vasconcellos, C. Arnold, A. Nowak, E. Galopin, I. Sagnes, L. Lanco, A. Lemaître, and P. Senellart, Nat. Commun. 4, 1425 (2013).

[10] Y.-J. Wei et al., Nano Lett. 14, 6515 (2014).

[11] X. Ding, Y. He, Z. C. Duan, N. Gregersen, M. C. Chen, S. Unsleber, S. Maier, C. Schneider, M. Kamp, S. Hofling, C. Y. Lu, and J. W. Pan, Phys. Rev. Lett. 116, 020401 (2016).

[12] S. Unsleber, Y.-M. He, S. Gerhardt, S. Maier, C.-Y. Lu, J.-Wei Pan, N. Gregersen, M. Kamp, C. Schneider, and S. Höfling, Opt. Express 24, 8539 (2016).

[13] N. Somaschi et al., Nat. Photonics 10, 340 (2016).

[14] J.-W. Pan, Z.-Bing Chen, C.-Y. Lu, H. Weinfurter, A. Zeilinger, and M. Żukowski, Rev. Mod. Phys. 84, 777 (2012).

[15] S. Aaronson and A. Arkhipov, in Proceedings of the 43rd Annual ACM Symposium on Theory of Computing, San Jose, 2011 (ACM, New York, 2011), p. 333.

[16] X.-L. Wang, X.-D. Cai, Z.-E. Su, M.-C. Chen, D. Wu, L. Li, N.-L. Liu, C.-Y. Lu, and J.-W. Pan, Nature (London) 518, 516 (2015).

[17] P. Kok, W. J. Munro, K. Nemoto, T. C. Ralph, J. P. Dowling, and G. J. Milburn, Rev. Mod. Phys. 79, 135 (2007); J. L. O’Brien, A. Furusawa, and J. Vuĉković, Nat. Photonics 3, 687 (2009).

[18] V. Giovannetti, S. Lloyd, and L. Maccone, Science 306, 1330 (2004); J. P. Dowling, Contemp. Phys. 49, 125 (2008).

[19] E. B. Flagg, A. Muller, S. V. Polyakov, A. Ling, A. Migdall, and G. S. Solomon, Phys. Rev. Lett. 104, 137401 (2010); Y. He, Y. M. He, Y. J. Wei, X. Jiang, M. C. Chen, F. L. Xiong, Y. Zhao, C. Schneider, M. Kamp, S. Hofling, C. Y. Lu, and J. W. Pan, Phys. Rev. Lett. 111, 237403 (2013).

[20] R. B. Patel, A. J. Bennett, I. Farrer, C. A. Nicoll, D. A. Ritchie, and A. J. Shields, Nat. Photonics 4, 632 (2010).

[21] P. C. Humphreys, B. J. Metcalf, J. B. Spring, M. Moore, X. M. Jin, M. Barbieri, W. S. Kolthammer, and I. A. Walmsley, Phys. Rev. Lett. 111, 150501 (2013); K. R. Motes, A. Gilchrist, J. P. Dowling, and P. P. Rohde, Phys. Rev. Lett. 113, 120501 (2014).

[22] A. V. Kuhlmann, J. Houel, A. Ludwig, L. Greuter, D. Reuter, A. D. Wieck, M. Poggio, and R. J. Warburton, Nat. Phys. 9, 570 (2015).

[23] C. Matthiesen, A. N. Vamivakas, and M. Atatüre, Phys. Rev. Lett. 108, 093602 (2012); C. Matthiesen, M. Geller, C. H. H. Schulte, C. Le Gall, J. Hansom, Z. Li, M. Hugues, E. Clarke, and M. Atatüre, Nat. Commun. 4, 1600 (2013).

[24] A. Muller, E. B. Flagg, P. Bianucci, X. Y. Wang, D. G. Deppe, W. Ma, J. Zhang, G. J. Salamo, M. Xiao, and C. K. Shih, Phys. Rev. Lett. 99, 187402 (2007); A. N. Vamivakas, Y. Zhao, C.-Y. Lu, and M. Atatüre, Nat. Phys. 5, 198 (2009); E. B. Flagg, A. Muller, J. W. Robertson, S. Founta, D. G. 
Deppe, M. Xiao, W. Ma, G. J. Salamo, and C. K. Shih, Nat. Phys. 5, 203 (2009).

[25] A. Delteil, Zhe Sun, W.-b. Gao, E. Togan, S. Faelt, and A. Imamoğlu, Nat. Phys. 12, 218 (2016).

[26] A. V. Kuhlmann, J. H. Prechtel, J. Houel, A. Ludwig, D. Reuter, A. D. Wieck, and R. J. Warburton, Nat. Commun. 6, 8204 (2015).

[27] A. Thoma, P. Schnauber, M. Gschrey, M. Seifried, J. Wolters, J. H. Schulze, A. Strittmatter, S. Rodt, A. Carmele, A. Knorr, T. Heindel, and S. Reitzenstein, Phys. Rev. Lett. 116, 033601 (2016).

[28] The wafer is grown via molecular beam epitaxy on a silicon doped GaAs wafer. On top of a $400 \mathrm{~nm}$ thick, intrinsic GaAs buffer layer, 25.5 (15) AlAs/GaAs mirror pairs, which form the lower (upper) distributed Bragg reflector, are grown. The distributed Bragg reflector stacks sandwich a $\lambda$-thick GaAs cavity with a single, low density layer of $\operatorname{In}(\mathrm{Ga}) \mathrm{As}$ QDs as the active medium. The partial capping and annealing technique is applied to tune the QD emission wavelength to $\sim 900 \mathrm{~nm}$. Underneath the QD layer, we implement a silicon $\delta$-doped layer with a dopant density of approximately $10^{9} / \mathrm{cm}^{2}$. After the growing is completed, micropillars with varying diameters ranging from 1 to $8 \mu \mathrm{m}$ are defined via electron beam lithography and transferred into the sample by electron-cyclotron-resonance reactive ion etching. The sample is then planarized by the polymer benzocyclobutene and the etch mask is fully removed.

[29] R. Hanbury Brown and R. Q. Twiss, Nature (London) 178, 1447 (1956).
[30] S. Unsleber, C. Schneider, S. Maier, Y.-M. He, S. Gerhardt, C.-Y. Lu, J.-W. Pan, M. Kamp, and S. Höfling, Opt. Express 23, 32977 (2015).

[31] See Supplemental Material at http://link.aps.org/ supplemental/10.1103/PhysRevLett.116.213601 for the data figures on the measurement of micropillar cavity and photon indistinguishability under different conditions.

[32] C. K. Hong, Z. Y. Ou, and L. Mandel, Phys. Rev. Lett. 59, 2044 (1987).

[33] L. Monniello, C. Tonin, R. Hostein, A. Lemaitre, A. Martinez, V. Voliotis, and R. Grousson, Phys. Rev. Lett. 111, 026403 (2013).

[34] A. J. Bennett, D. C. Unitt, A. J. Shields, P. Atkinson, and D. A. Ritchie, Opt. Express 13, 7772 (2005); E. B. Flagg, A. Muller, S. V. Polyakov, A. Ling, A. Migdall, and G. S. Solomon, Phys. Rev. Lett. 104, 137401 (2010).

[35] C. Santori, D. Fattal, J. Vuckovic, G. S Solomon, and Y. Yamamoto, New J. Phys. 6, 89 (2004); S. Unsleber, D. P. S. McCutcheon, M. Dambach, M. Lermer, N. Gregersen, S. Hofling, J. Mork, C. Schneider, and M. Kamp, Phys. Rev. B 91, 075413 (2015).

[36] A. J. Ramsay, A. V. Gopal, E. M. Gauger, A. Nazir, B. W. Lovett, A. M. Fox, and M. S. Skolnick, Phys. Rev. Lett. 104, 017402 (2010); A. J. Ramsay, T. M. Godden, S. J. Boyle, E. M. Gauger, A. Nazir, B. W. Lovett, A. M. Fox, and M. S. Skolnick, Phys. Rev. Lett. 105, 177402 (2010).

[37] P. Kaer and J. Mørk, Phys. Rev. B 90, 035312 (2014).

[38] Y. He et al., arXiv:1603.04127.

[39] J. C. Loredo et al., Optica 3, 433 (2016). 